\title{
Conservation Laws and Normal Forms of Evolution Equations
}

\author{
Roman O. POPOVYCH ${ }^{\dagger}$ and Artur SERGYEYEV $\ddagger$ \\ ${ }^{\dagger}$ Fakultät für Mathematik, Universität Wien, Nordbergstraße 15, A-1090 Wien, Austria \\ Institute of Mathematics of NAS of Ukraine, 3 Tereshchenkivska Str., Kyiv-4, Ukraine \\ ‡ Mathematical Institute, Silesian University in Opava, Na Rybničku 1, 74601 Opava, \\ Czech Republic \\ E-mail: ${ }^{\dagger}$ rop@imath.kiev.ua, ${ }^{\ddagger}$ Artur.Sergyeyev@math.slu.cz
}

\begin{abstract}
We study local conservation laws for evolution equations in two independent variables. In particular, we present normal forms for the equations admitting one or two low-order conservation laws. Examples include Harry Dym equation, Korteweg-de-Vries-type equations, and Schwarzian KdV equation. It is also shown that for linear evolution equations all their conservation laws are (modulo trivial conserved vectors) at most quadratic in the dependent variable and its derivatives.
\end{abstract}

\section{Introduction}

The role played in the sciences by linear and nonlinear evolution equations and, in particular, by conservation laws thereof, is hard to overestimate (recall e.g. linear and nonlinear Schrödinger equations and the Korteweg-de Vries (KdV) equation in physics, reaction-diffusion systems in chemistry and biology, and the Black-Scholes equation in the finance, to name just a few). For instance, the discovery of higher conservation laws for the $\mathrm{KdV}$ equations provided an important milestone on the way that has eventually lead to the discovery of the inverse scattering transform and the modern theory of integrable systems, see e.g. [21, 22]. However, the theory of conservation laws for evolution equations is still far from being complete even for the simplest case of two independent variables, and in the present paper we address some issues of the theory in question for this very case.

We shall deal with an evolution equation in two independent variables,

$$
u_{t}=F\left(t, x, u_{0}, u_{1}, \ldots, u_{n}\right), \quad n \geq 2, \quad F_{u_{n}} \neq 0
$$

where $u_{j} \equiv \partial^{j} u / \partial x^{j}, u_{0} \equiv u$, and $F_{u_{j}}=\partial F / \partial u_{j}$. We shall also employ, depending on convenience or necessity, the following notation for low-order derivatives: $u_{x}=u_{1}, u_{x x}=u_{2}$, and $u_{x x x}=u_{3}$.

There is a considerable body of results on conservation laws of evolution equations of the form (11). For instance, in the seminal paper [8] the authors studied, inter alia, conservation laws of Eq. (11) with $\partial F / \partial t=0$ for $n=2$. They proved that the possible dimensions of spaces of inequivalent conservation laws for such equations are $0,1,2$ and $\infty$, and described the equations possessing spaces of conservation laws of these dimensions (the precise definitions of equivalence and order of conservation laws are given in the next section). These results were further generalized in [28] for the case when $F$ explicitly depends on $t$.

Important results on conservation laws of (11), typically under the assumptions of polynomiality and $t, x$-independence of $F$ and of the conservation laws themselves, were obtained in [1, 2, 3, 4, 10, 11, 12, 15. However, for general Eq. (11) there is no simple picture analogous to that of the second-order case discussed above. For instance, unlike the second-order case, there exist odd-order evolution equations that possess infinitely many inequivalent conservation laws of increasing orders without being linearizable. Rather, such equations are integrable via 
the inverse scattering transform, the famous $\mathrm{KdV}$ equation providing a prime example of such behavior, see e.g. [13, 15, 21] and references therein; for the fifth-order equations see [9].

Note that many results on symmetries and conservation laws were obtained using the formal symmetry approach and modifications thereof, see e.g. the recent survey [20] and references therein, in particular [19, 34]. For instance, it was shown that an equation (1) of even order $(n=$ $2 m$ ) has no conservation laws (modulo trivial ones) of order greater than $m$, see [1, 10, 13, 14] for details. There also exists a closely related approach to the study of symmetries and conservation laws of evolution equations, the so-called symbolic method, see [18, 29, 30, 31] and references therein for details.

However, many important questions concerning the conservation laws of evolution equations were not answered so far. For example, we are not aware of any significant advances in the study of normal forms of evolution equations admitting low-order conservation laws considered in $[8,11,28]$. In the present paper we provide such normal forms with respect to contact or point transformations for equations admitting one or two low-order conservation laws, respectively, see Theorems 1 and 2 below. Let us stress that in what follows we restrict ourselves to considering only local conservation laws whose densities and fluxes depend only on the independent and dependent variables and a finite number of the derivatives of the latter.

The complete description of conservation laws for linear evolution equations with $t, x$ dependent coefficients was also missing so far. Below we show that linear even-order equations of the form (11) can only possess conservation laws linear in $u_{j}$ for all $j=0,1,2, \ldots$ while the odd-order equations can further admit the conservation laws (at most) quadratic in $u_{j}$, see Theorems 3 and 4 , Corollary 6 and Theorem 5 below. This naturally generalizes some earlier results from [3, 12]; cf. also [5]. The generation of linear and quadratic conservation laws for linear differential equations is also discussed in some depth in [24, Section 5.3].

Below we denote by $\mathrm{CL}(\mathcal{E})$ the space of local conservation laws of $\mathcal{E}$ (cf. Section 3 ), where $\mathcal{E}$ denotes a fixed equation from the class (1). In what follows $D_{t}$ and $D_{x}$ stand for the total derivatives (see e.g. 24] for details) with respect to the variables $t$ and $x$,

$$
D_{t}=\partial_{t}+u_{t} \partial_{u}+u_{t t} \partial_{u_{t}}+u_{t x} \partial_{u_{x}}+\cdots, \quad D_{x}=\partial_{x}+u_{x} \partial_{u}+u_{t x} \partial_{u_{t}}+u_{x x} \partial_{u_{x}}+\cdots .
$$

As usual, the subscripts like $t, x, u, u_{x}$, etc. stand for the partial derivatives in the respective variables.

\section{Admissible transformations of evolution equations}

The contact transformations mapping an equation from class (11) into another equation from the same class are well known [17] to have the form

$$
\tilde{t}=T(t), \quad \tilde{x}=X\left(t, x, u, u_{x}\right), \quad \tilde{u}=U\left(t, x, u, u_{x}\right) .
$$

The functions $T, X$ and $U$ must satisfy the nondegeneracy assumptions, namely, $T_{t} \neq 0$ and

$$
\operatorname{rank}\left(\begin{array}{ccc}
X_{x} & X_{u} & X_{u_{x}} \\
U_{x} & U_{u} & U_{u_{x}}
\end{array}\right)=2
$$

and the contact condition

$$
\left(U_{x}+U_{u} u_{x}\right) X_{u_{x}}=\left(X_{x}+X_{u} u_{x}\right) U_{u_{x}} .
$$

The transformation (2) is uniquely extended to the derivative $u_{x}$ and to the higher derivatives by the formulas $\tilde{u}_{\tilde{x}}=V\left(t, x, u, u_{x}\right)$ and $\tilde{u}_{k} \equiv \partial^{k} \tilde{u} / \partial \tilde{x}^{k}=\left(\left(1 / D_{x} X\right) D_{x}\right)^{k} V$, where

$$
V=\frac{U_{x}+U_{u} u_{x}}{X_{x}+X_{u} u_{x}} \quad \text { or } \quad V=\frac{U_{u_{x}}}{X_{u_{x}}}
$$


if $X_{x}+X_{u} u_{x} \neq 0$ or $X_{u_{x}} \neq 0$, respectively; the possibility of simultaneous vanishing of these two quantities is ruled out by (3).

The transformed equation (11) reads $\tilde{u}_{\tilde{t}}=\tilde{F}$ where

$$
\tilde{F}=\frac{U_{u}-X_{u} V}{T_{t}} F+\frac{U_{t}-X_{t} V}{T_{t}}
$$

and $\left(X_{u}, U_{u}\right) \neq(0,0)$ because of (3) and (4)).

Any transformation of the form (2) leaves the class (11) invariant, and therefore its extension to an arbitrary element $F$ belongs to the contact equivalence group $G_{\mathrm{c}}^{\sim}$ of class (11), so there are no other elements in $G_{\mathrm{c}}^{\sim}$. In other words, the equivalence group $G_{\mathrm{c}}^{\sim}$ generates the whole set of admissible contact transformations in the class (11), i.e., this class is normalized with respect to contact transformations, see [26] for details.

The above results can be summarized as follows.

Proposition 1. The class of equations (11) is contact-normalized. The contact equivalence group $G_{\mathrm{c}}^{\sim}$ of the class (11) is formed by the transformations (22), satisfying conditions (3) and (4) and prolonged to the arbitrary element $F$ by (5).

Furthermore, the class (1) is also point-normalized. The point equivalence group $G_{\mathrm{p}}^{\sim}$ of this class consists of the transformations of the form

$$
\tilde{t}=T(t), \quad \tilde{x}=X(t, x, u), \quad \tilde{u}=U(t, x, u), \quad \tilde{F}=\frac{\Delta}{T_{t} D_{x} X} F+\frac{U_{t} D_{x} X-X_{t} D_{x} U}{T_{t} D_{x} X},
$$

where $T, X$ and $U$ are arbitrary smooth functions that satisfy the nondegeneracy conditions $T_{t} \neq 0$ and $\Delta=X_{x} U_{u}-X_{u} U_{x} \neq 0$.

Notice that there exist subclasses of the class (1) whose sets of admissible contact transformations are exhausted by point transformations.

In the present paper we do not consider more general transformations, e.g., differential substitutions such as the Cole-Hopf transformation.

\section{Some basic results on conservation laws}

It is well known that for any evolution equation (1) we can assume without loss of generality that the associated quantities like symmetries, cosymmetries, densities, etc., can be taken to be independent of the $t$-derivatives or mixed derivatives of $u$. We shall stick to this assumption throughout the rest of the paper.

Following [24] we shall refer to a (smooth) function of $t, x$ and a finite number of $u_{j}$ as to a differential function. Given a differential function $f$, its order (denoted by ord $f$ ) is the greatest integer $k$ such that $f_{u_{k}} \neq 0$ but $f_{u_{j}}=0$ for all $j>k$. For $f=f(t, x)$ we assume that ord $f=0$.

Thus, for a (fixed) evolution equation (1), which we denote by $\mathcal{E}$ as before, we lose no generality [24] in considering only the conserved vectors of the form $(\rho, \sigma)$, where $\rho$ and $\sigma$ are differential functions which satisfy the condition

$$
D_{t} \rho+D_{x} \sigma=0 \bmod \check{\mathcal{E}},
$$

and $\check{\mathcal{E}}$ means the equation $\mathcal{E}$ together with all its differential consequences. Here $\rho$ is the density and $\sigma$ is the flux for the conserved vector $(\rho, \sigma)$. Let

$$
\frac{\delta}{\delta u}=\sum_{i=0}^{\infty}\left(-D_{x}\right)^{i} \partial_{u_{i}}, \quad f_{*}=\sum_{i=0}^{\infty} f_{u_{i}} D_{x}^{i}, \quad f_{*}^{\dagger}=\sum_{i=0}^{\infty}\left(-D_{x}\right)^{i} \circ f_{u_{i}}
$$


denote the operator of variational derivative, the Fréchet derivative of a differential function $f$, and its formal adjoint, respectively. With this notation in mind we readily infer that the condition (7) can be rewritten as $\rho_{t}+\rho_{*} F+D_{x} \sigma=0$. As $\rho_{*} F=F \delta \rho / \delta u+D_{x} \zeta$ for some differential function $\zeta$, see e.g. [13, Section 22.5], there exists a differential function $\Psi$ (in fact, $\Psi=-\zeta-\sigma$ ) such that

$$
\rho_{t}+F \frac{\delta \rho}{\delta u}=D_{x} \Psi
$$

A conserved vector $(\rho, \sigma)$ is called trivial if it satisfies the condition $D_{t} \rho+D_{x} \sigma=0$ on the entire jet space. It is easily seen that the conserved vector $(\rho, \sigma)$ is trivial if and only if $\rho \in \operatorname{Im} D_{x}$, i.e., there exists a differential function $\zeta$ such that $\rho=D_{x} \zeta$. Two conserved vectors are equivalent if they differ by a trivial conserved vector. We shall call a conservation law of $\mathcal{E}$ an equivalence class of conserved vectors of $\mathcal{E}$. The set $\mathrm{CL}(\mathcal{E})$ of conservation laws of $\mathcal{E}$ is a vector space, and the zero element of this space is the conservation law being the equivalence class of trivial conserved vectors. This is why nonzero conservation laws are usually called nontrivial.

For any conservation law $\mathcal{L}$ of $\mathcal{E}$ there exists a unique differential function $\gamma$ called the characteristic of $\mathcal{L}$ such that for any conserved vector $(\rho, \sigma)$ associated with $\mathcal{L}$ (we shall write this as $(\rho, \sigma) \in \mathcal{L})$ there exists a trivial conserved vector $(\tilde{\rho}, \tilde{\sigma})$ satisfying the condition

$$
D_{t}(\rho+\tilde{\rho})+D_{x}(\sigma+\tilde{\sigma})=\gamma\left(u_{t}-F\right)
$$

It is important to stress that, unlike (7), the above equation holds on the entire jet space rather than merely modulo $\check{\mathcal{E}}$.

The characteristic $\gamma$ of any conservation law satisfies the equation (see e.g. [24])

$$
D_{t} \gamma+F_{*}^{\dagger} \gamma=0 \bmod \check{\mathcal{E}}, \quad \text { or equivalently, } \quad \gamma_{t}+\gamma_{*} F+F_{*}^{\dagger} \gamma=0
$$

However, in general a solution of (10) is not necessarily a characteristic of some conservation law for (11). Solutions of (10) are called cosymmetries, see e.g. [6].

It can be shown that the characteristic of the conservation law associated with a conserved vector of the form $(\rho, \sigma)$ equals $\delta \rho / \delta u$. This yields a necessary and sufficient condition for a cosymmetry $\gamma$ to be a characteristic of a conservation law (see e.g. [24]): $\gamma_{*}=\gamma_{*}^{\dagger}$. This condition means simply that the Fréchet derivative of $\gamma$ is formally self-adjoint.

The following results are well known, see e.g. 10 for Lemma 2

Lemma 1. Suppose that an equation from the class (11) admits a nontrivial conserved vector $(\rho, \sigma)$, where ord $\rho=k>0$, and $\rho_{u_{k} u_{k}}=0$. Then the conserved vector $(\rho, \sigma)$ is equivalent to a conserved vector $(\tilde{\rho}, \tilde{\sigma})$ with ord $\tilde{\rho} \leqslant k-1$.

Proof. By assumption, $\rho=\rho^{1} u_{k}+\rho^{0}$, and hence $\sigma=-\rho^{1} D_{x}^{k-1}(F)+\sigma^{0}$, where $\rho^{1}=$ $\rho^{1}\left(t, x, u, u_{1}, \ldots, u_{k-1}\right), \rho^{0}=\rho^{0}\left(t, x, u, u_{1}, \ldots, u_{k-1}\right)$, and $\sigma^{0}=\sigma^{0}\left(t, x, u, u_{1}, \ldots, u_{k+n}\right)$. Put $\tilde{\rho}=\rho-D_{x} \Phi$ and $\tilde{\sigma}=\sigma+D_{t} \Phi$, where $\Phi=\int \rho^{1} d u_{k-1}$. Then $\tilde{\rho}_{u_{k}}=0, \tilde{\sigma}_{u_{k+n}}=0$ and $(\tilde{\rho}, \tilde{\sigma})$ is a conserved vector equivalent to $(\rho, \sigma)$, and ord $\tilde{\rho} \leqslant k-1$.

In what follows, for any given conservation law $\mathcal{L}$ we shall, unless otherwise explicitly stated, choose a representative (that is, the conserved vector) with the lowest possible order $k$ of the associated density $\rho$. The order in question (i.e., the greatest integer $k$ such that $\rho_{u_{k} u_{k}} \neq 0$ but $\rho_{u_{j}}=0$ for all $j>k$ ) will be called the density order of $\mathcal{L}$ and denoted by $\operatorname{ord}_{\mathrm{d}} \mathcal{L}$. It equals one half of the order of the associated characteristic.

Lemma 2. For any conservation law $\mathcal{L}$ of a $(1+1)$-dimensional even-order $(n=2 q)$ evolution equation of the form (11) we have $\operatorname{ord}_{\mathrm{d}} \mathcal{L} \leqslant q$. 


\section{Evolution equations having low-order conservation laws}

Contact and point equivalence transformations can be used for bringing equations from the class (11) that admit (at least) one or two nontrivial low-order conservation laws into certain special forms. This is achieved through bringing the conservation laws in question to normal forms.

Theorem 1. Any pair $(\mathcal{E}, \mathcal{L})$, where $\mathcal{E}$ is an equation of the form (11) and $\mathcal{L}$ is a nontrivial conservation law of $\mathcal{E}$ with $\operatorname{ord}_{\mathrm{d}} \mathcal{L} \leqslant 1$ is $G_{\mathrm{c}}^{\sim}$-equivalent to a pair $(\tilde{\mathcal{E}}, \tilde{\mathcal{L}})$, where $\tilde{\mathcal{E}}$ is an equation of the same form and $\tilde{\mathcal{L}}$ is a conservation law of $\tilde{\mathcal{E}}$ with the characteristic equal to 1.

Proof. Let $\mathcal{T} \in G_{\mathrm{c}}^{\sim}$ map an equation $\mathcal{E}$ into (another) equation $\tilde{\mathcal{E}}$ from the same class (11), see Section 2, Quite obviously, the inverse $\mathcal{T}^{-1}$ of $\mathcal{T}$ induces (through pullback) a mapping from the space $\mathrm{CL}(\mathcal{E})$ of conservation laws of $\mathcal{E}$ to $\mathrm{CL}(\tilde{\mathcal{E}})$. The conserved vectors of $\mathcal{E}$ are transformed into those of $\tilde{\mathcal{E}}$ according to the formula [25, 27]

$$
\tilde{\rho}=\frac{\rho}{D_{x} X}, \quad \tilde{\sigma}=\frac{\sigma}{T_{t}}+\frac{D_{t} X}{D_{x} X} \frac{\rho}{T_{t}} .
$$

Now let an equation $\mathcal{E}$ from the class (11) have a nontrivial conservation law $\mathcal{L}$ with $\operatorname{ord}_{\mathrm{d}} \mathcal{L} \leqslant 1$. Fix a conserved vector $(\rho, \sigma)$ associated with $\mathcal{L}$, and set $T=t$. The density $\tilde{\rho}$ of the transformed conserved vector $(\tilde{\rho}, \tilde{\sigma})$ is easily seen to depend at most on $\tilde{t}, \tilde{x}, \tilde{u}, \tilde{u}_{\tilde{x}}$ and $\tilde{u}_{\tilde{x} \tilde{x}}$. Moreover, it is immediate that $\tilde{\rho}$ is linear in $\tilde{u}_{\tilde{x} \tilde{x}}$, so we can pass to an equivalent conserved vector $(\bar{\rho}, \bar{\sigma})$ such that $\partial \bar{\rho} / \partial \tilde{u}_{\tilde{x}} \tilde{x}=0$, and hence for the transformed counterpart $\tilde{\mathcal{L}}$ of $\mathcal{L}$ we have $\operatorname{ord}_{\mathrm{d}} \tilde{\mathcal{L}} \leqslant 1$.

Next, the conservation law $\tilde{\mathcal{L}}$ associated with $(\tilde{\rho}, \tilde{\sigma})$ has characteristic 1 if and only if there exists a function $\tilde{\Phi}=\tilde{\Phi}\left(\tilde{t}, \tilde{x}, \tilde{u}, \tilde{u}_{\tilde{x}}\right)$ such that $\tilde{\rho}=\tilde{u}+D_{\tilde{x}} \tilde{\Phi}$. Upon going back to the old coordinates $x, t, u, u_{x}$ and bearing in mind that $\tilde{u}=U\left(t, x, u, u_{x}\right)$ and $\tilde{x}=X\left(t, x, u, u_{x}\right)$ this boils down to $D_{x} \Phi+U D_{x} X=\rho$, where $\Phi\left(t, x, u, u_{x}\right)=\tilde{\Phi}\left(\tilde{t}, \tilde{x}, \tilde{u}, \tilde{u}_{\tilde{x}}\right)$. Splitting the equation $D_{x} \Phi+U D_{x} X=\rho$ with respect to $u_{x x}$ yields the system

$$
\Phi_{x}+U X_{x}+\left(\Phi_{u}+U X_{u}\right) u_{x}=\rho, \quad \Phi_{u_{x}}+U X_{u_{x}}=0 .
$$

This system in conjunction with the contact condition (4) has, inter alia, the following differential consequence:

$$
\Phi_{u}+U X_{u}=\rho_{u_{x}} .
$$

It is obtained as follows. We subtract the result of action of the operator $\partial_{x}+u_{x} \partial_{u}$ on the second equation of (11) from the partial $u_{x}$-derivative of the first equation of (11) while taking into account the contact condition (4). Moreover, the system (11) also implies the equation $\Phi_{x}+U X_{x}=\rho-u_{x} \rho_{u_{x}}$. Thus, we arrive at the system

$$
\Phi_{x}+U X_{x}=\rho-u_{x} \rho_{u_{x}}, \quad \Phi_{u}+U X_{u}=\rho_{u_{x}}, \quad \Phi_{u_{x}}+U X_{u_{x}}=0 .
$$

Reversing these steps shows that the system (12) implies (41) and (11). Hence the combined system of (41) and (11) is equivalent to (12).

To complete the proof, it suffices to check that for any function $\rho=\rho\left(t, x, u, u_{x}\right)$ with $\left(\rho_{u}, \rho_{u_{x}}\right) \neq(0,0)$ the system (12) has a solution $(X, U, \Phi)$ which satisfies the nondegeneracy condition (3).

Consider first the case $\rho_{u_{x} u_{x}} \neq 0$ and seek for solutions with $X_{u_{x}} \neq 0$. The equation $\Phi_{u_{x}}+$ $U X_{u_{x}}=0$ implies that $\Phi_{u_{x}} \neq 0$ and $U=-\Phi_{u_{x}} / X_{u_{x}}$. Then the remaining equations in (12) take the form

$$
\Phi_{x}-\frac{X_{x}}{X_{u_{x}}} \Phi_{u_{x}}=\rho-u_{x} \rho_{u_{x}}, \quad \Phi_{u}-\frac{X_{u}}{X_{u_{x}}} \Phi_{u_{x}}=\rho_{u_{x}}
$$


Eq. (13) can be considered as an overdetermined system with respect to $\Phi$. The compatibility condition for this system is

$$
\rho_{u_{x} u_{x}} X_{x}+u_{x} \rho_{u_{x} u_{x}} X_{u}+\left(\rho_{u}-u_{x} \rho_{u u_{x}}-\rho_{x u_{x}}\right) X_{u_{x}}=0
$$

it should be treated as an equation for $X$. As $\rho_{u_{x} u_{x}} \neq 0$ by assumption, the equation in question has a local solution $X^{0}$ with $X_{u_{x}}^{0} \neq 0$. Substituting $X^{0}$ into (13) yields a compatible partial differential system for $\Phi$. Take a local solution $\Phi^{0}$ of this system and set $U^{0}=-\Phi_{u_{x}}^{0} / X_{u_{x}}^{0}$. The chosen triple $\left(X^{0}, U^{0}, \Phi^{0}\right)$ satisfies (12).

The nondegeneracy condition (3) is also satisfied. Indeed, if we assume the converse, then $U=\Psi(t, X)$ for some function $\Psi$ of two arguments, and (11) implies the equality

$$
\rho=\Phi_{x}+\Psi X_{x}+\left(\Phi_{u}+\Psi X_{u}\right) u_{x}+\left(\Phi_{u_{x}}+\Psi X_{u_{x}}\right) u_{x x}=D_{x}\left(\Phi+\int \Psi d X\right),
$$

i.e., $(\rho, \sigma)$ is a trivial conserved vector, which contradicts the initial assumption on $(\rho, \sigma)$.

Now turn to the case when $\rho_{u_{x} u_{x}}=0$. Then up to the equivalence of conserved vectors we can assume that $\rho_{u_{x}}=0$ and $\rho_{u} \neq 0$, where the latter condition ensures nontriviality of the associated conserved vector. The triple $(X, U, \Phi)=(x, \rho, 0)$ obviously satisfies (12) and (3) , and the result follows.

Corollary 1. Any pair $(\mathcal{E}, \mathcal{L})$, where $\mathcal{E}$ is an equation of the form (1) and $\mathcal{L}$ is a nontrivial conservation law of $\mathcal{E}$ with the density order 0 is $G_{\mathrm{p}}^{\sim}$-equivalent to a pair $(\tilde{\mathcal{E}}, \tilde{\mathcal{L}})$, where $\tilde{\mathcal{E}}$ also is an equation of form (1) and $\tilde{\mathcal{L}}$ is a conservation law of $\tilde{\mathcal{E}}$ with the characteristic equal to 1.

Corollary 2. An equation $\mathcal{E}$ from class (1) admits a nontrivial conservation law $\mathcal{L}$ with $\operatorname{ord}_{\mathrm{d}} \mathcal{L} \leqslant 1$ (resp. $\operatorname{ord}_{\mathrm{d}} \mathcal{L}=0$ ) if and only if it can be locally reduced by a contact (resp. point) transformation to the form

$$
\tilde{u}_{\tilde{t}}=D_{\tilde{x}} G\left(\tilde{t}, \tilde{x}, \tilde{u}_{0}, \ldots, \tilde{u}_{n-1}\right), \quad G_{\tilde{u}_{n-1}} \neq 0 .
$$

Note that upon setting $n=3$ and $\operatorname{ord}_{\mathrm{d}} \mathcal{L}=0$ in this corollary we recover Theorem 1.1 from [1].

Proof. Fix a nontrivial conservation law $\mathcal{L}$ of $\mathcal{E}$ with $\operatorname{ord}_{\mathrm{d}} \mathcal{L} \leqslant 1\left(\operatorname{resp} \operatorname{ord}_{\mathrm{d}} \mathcal{L}=0\right)$. By Theorem 1 (resp. Corollary 1), the pair $(\mathcal{E}, \mathcal{L})$ is reduced by a contact (resp. point) transformation to a pair $(\tilde{\mathcal{E}}, \tilde{\mathcal{L}})$, where the equation $\tilde{\mathcal{E}}$ has the form $\tilde{u}_{\tilde{t}}=\tilde{F}\left(\tilde{t}, \tilde{x}, \tilde{u}_{0}, \ldots, \tilde{u}_{n}\right)$ and $\tilde{\mathcal{L}}$ is its conservation law with the unit characteristic. Therefore, the equality $D_{\tilde{t}} \tilde{\rho}+D_{\tilde{x}} \tilde{\sigma}=\tilde{u}_{\tilde{t}}-\tilde{F}$ is satisfied for a conserved vector $(\tilde{\rho}, \tilde{\sigma})$ from $\tilde{\mathcal{L}}$, i.e., up to a summand being a null divergence we have $\tilde{\rho}=\tilde{u}$ and $\tilde{F}=-D_{\tilde{x}} \tilde{\sigma}$. To complete the proof, it suffices to put $G=-\tilde{\sigma}$.

Conversely, let the equation $\mathcal{E}$ be locally reducible by a contact (resp. point) transformation $\mathcal{T}$ to the equation $\tilde{u}_{\tilde{t}}=D_{\tilde{x}} G\left(\tilde{t}, \tilde{x}, \tilde{u}_{0}, \ldots, \tilde{u}_{n-1}\right)$, where $G_{\tilde{u}_{n-1}} \neq 0$. The transformed equation $\tilde{u}_{\tilde{t}}=D_{\tilde{x}} G$ admits at least the conservation law $\tilde{\mathcal{L}}$ with the unit characteristic. The preimage $\mathcal{L}$ of $\tilde{\mathcal{L}}$ with respect to $\mathcal{T}$ is a nontrivial conservation law of $\mathcal{E}$ with $\operatorname{ord}_{\mathrm{d}} \mathcal{L} \leqslant 1\left(\operatorname{resp}\right.$. $\left.\operatorname{ord}_{\mathrm{d}} \mathcal{L}=0\right)$.

Corollary 3. If an equation $\mathcal{E}$ of the form (1) with $n \geqslant 4$ (resp. $n \geqslant 5$ ) has two linearly independent conservation laws $\mathcal{L}^{\mathrm{I}}$ and $\mathcal{L}^{\mathrm{II}}$, where $\operatorname{ord}_{\mathrm{d}} \mathcal{L}^{\mathrm{I}} \leqslant 1$ and $\operatorname{ord}_{\mathrm{d}} \mathcal{L}^{\mathrm{II}} \leqslant n / 2-1$ (resp. $\left.\operatorname{ord}_{\mathrm{d}} \mathcal{L}^{\mathrm{II}}<n / 2-1\right)$ then it can be locally reduced by a contact transformation to the form (14) where $G$ is linear fractional (resp. linear) with respect to $\tilde{u}_{n-1}$, i.e.,

$$
G=\frac{G_{1} \tilde{u}_{n-1}+G_{0}}{G_{3} \tilde{u}_{n-1}+G_{2}} \quad\left(\text { resp. } G=G_{1} \tilde{u}_{n-1}+G_{0}\right),
$$

where $G_{0}, \ldots, G_{3}$ (resp. $G_{0}$ and $G_{1}$ ) are differential functions of order less than $n-1$. If $\operatorname{ord}_{\mathrm{d}} \mathcal{L}^{\mathrm{I}}=0$ then the contact transformation in question is a prolongation of a point transformation. 
Proof. Without loss of generality we can assume that $\operatorname{ord}_{\mathrm{d}} \mathcal{L}^{\mathrm{I}} \leqslant \operatorname{ord}_{\mathrm{d}} \mathcal{L}^{\mathrm{II}}$. By Theorem 1 and Corollary 2, the pair $\left(\mathcal{E}, \mathcal{L}^{\mathrm{I}}\right)$ is reduced by a contact transformation to a pair $\left(\tilde{\mathcal{E}}, \tilde{\mathcal{L}}^{\mathrm{I}}\right)$, where the equation $\tilde{\mathcal{E}}$ is of form (14) and the conservation law $\tilde{\mathcal{L}}^{\mathrm{I}}$ has the density $\tilde{u}$. The transformed conservation law $\tilde{\mathcal{L}}^{\mathrm{II}}$ satisfies the same inequality as the original one, $\mathcal{L}^{\mathrm{II}}$, i.e., $\operatorname{ord}_{\mathrm{d}} \tilde{\mathcal{L}}^{\mathrm{II}} \leqslant n / 2-1$ $\left(\right.$ resp. $\left.\operatorname{ord}_{\mathrm{d}} \tilde{\mathcal{L}}^{\mathrm{II}}<n / 2-1\right)$ if $n \geqslant 4$ (resp. $\left.n \geqslant 5\right)$. Below we omit tildes over the transformed variables for convenience and assume that the conservation law $\mathcal{L}^{\mathrm{I}}$ possesses the density $u$ and, therefore, the equation $\mathcal{E}$ has the form (14). Let $\left(\rho^{\mathrm{II}}, \sigma^{\mathrm{II}}\right)$ be a conserved vector associated with $\mathcal{L}^{\mathrm{II}}$ and $\operatorname{ord} \rho^{\mathrm{II}}=\operatorname{ord}_{\mathrm{d}} \mathcal{L}^{\mathrm{II}}$. By (8) , it satisfies the condition $\rho_{t}^{\mathrm{II}}+\left(D_{x} G\right) \delta \rho^{\mathrm{II}} / \delta u=D_{x} \Psi$ for some differential function $\Psi$. The last equality can be rewritten as

$$
\rho_{t}^{\mathrm{II}}-G D_{x}\left(\delta \rho^{\mathrm{II}} / \delta u\right)=D_{x} \Phi,
$$

where $\Phi=\Psi-G \delta \rho^{\mathrm{II}} / \delta u$. Note that $D_{x}\left(\delta \rho^{\mathrm{II}} / \delta u\right) \neq 0$ because otherwise the conservation laws $\mathcal{L}^{\mathrm{I}}$ and $\mathcal{L}^{\mathrm{II}}$ are linearly dependent. As ord $\rho_{t}^{\mathrm{II}}<n-1$, ord $G=n-1$ and ord $D_{x}\left(\delta \rho^{\mathrm{II}} / \delta u\right) \leqslant n-1$ (resp. ord $\left.D_{x}\left(\delta \rho^{\mathrm{II}} / \delta u\right)<n-1\right)$, we have ord $\Phi<n-1$. Finally, as $D_{x}\left(\delta \rho^{\mathrm{II}} / \delta u\right)$ must be linear in the highest-order $x$-derivative of $u$ it contains, expressing $G$ from (15) and taking into account the above inequalities for ord $D_{x}\left(\delta \rho^{\mathrm{II}} / \delta u\right)$ immediately yields the desired result.

Theorem 2. Let $\mathcal{E}$ be an equation of the form (1) and $\mathcal{L}^{\mathrm{I}}$ and $\mathcal{L}^{\mathrm{II}}$ be linearly independent conservation laws of $\mathcal{E}$ of density order 0. Any such triple $\left(\mathcal{E}, \mathcal{L}^{\mathrm{I}}, \mathcal{L}^{\mathrm{II}}\right)$ is $G_{\mathrm{p}}^{\sim}$-equivalent to a triple $\left(\tilde{\mathcal{E}}, \tilde{\mathcal{L}}^{\mathrm{I}}, \tilde{\mathcal{L}}^{\mathrm{II}}\right)$, where $\tilde{\mathcal{E}}$ is an equation from the same class (11) that admits conservation laws $\tilde{\mathcal{L}}^{\mathrm{I}}$ and $\tilde{\mathcal{L}^{\mathrm{II}}}$ with the characteristics equal to 1 and $\tilde{x}$, respectively.

Proof. Let $\left(\rho^{i}, \sigma^{i}\right) \in \mathcal{L}^{i}$ and ord $\rho^{i}=0, i=$ I, II. Then $\gamma^{i}=\rho_{u}^{i}$ is the characteristic of $\mathcal{L}^{i}$, $i=$ I, II. Moreover, $\gamma^{\mathrm{I}}$ and $\gamma^{\mathrm{II}}$ are linearly independent differential functions in view of the linear independence of conservation laws $\mathcal{L}^{\mathrm{I}}$ and $\mathcal{L}^{\mathrm{II}}$. Therefore, we have $\left(\lambda_{x}, \lambda_{u}\right) \neq(0,0)$, where $\lambda=\gamma^{\mathrm{II}} / \gamma^{\mathrm{I}}$. (Indeed, otherwise the substitution of these characteristics into (10) would imply that $\lambda_{t}=0$ as well, i.e., the characteristics $\gamma^{\mathrm{I}}$ and $\gamma^{\mathrm{II}}$ would be linearly dependent.)

We will prove the existence of (and, in fact, construct) a point equivalence transformation of the form (6) with $T(t)=t$ such that the transformed conserved vectors $\left(\tilde{\rho}^{\mathrm{I}}, \tilde{\sigma}^{\mathrm{I}}\right)$ and $\left(\tilde{\rho}^{\mathrm{II}}, \tilde{\sigma}^{\mathrm{II}}\right)$ are equivalent to the conserved vectors with the densities $\tilde{u}$ and $\tilde{x} \tilde{u}$, respectively. In other words, we want to have $\tilde{\rho}^{\mathrm{I}}=\tilde{u}+D_{\tilde{x}} \Phi$ and $\tilde{\rho}^{\mathrm{II}}=\tilde{x} \tilde{u}+D_{\tilde{x}} \Psi$ for some functions $\Phi=\Phi(t, x, u)$ and $\Psi=\Psi(t, x, u)$. In the old coordinates these conditions take the form $D_{x} \Phi+U D_{x} X=\rho^{\mathrm{I}}$ and $D_{x} \Psi+X U D_{x} X=\rho^{\mathrm{II}}$. Splitting them with respect to $u_{x}$ yields

$$
\begin{aligned}
& \Phi_{x}+U X_{x}=\rho^{\mathrm{I}}, \\
& \Phi_{u}+U X_{u}=0
\end{aligned} \quad \text { and } \quad \begin{aligned}
& \Psi_{x}+X U X_{x}=\rho^{\mathrm{II}}, \\
& \Psi_{u}+X U X_{u}=0
\end{aligned}
$$

After the elimination of $\Phi$ and $\Psi$ from these systems through cross-differentiation, we arrive at the conditions $X_{x} U_{u}-X_{u} U_{x}=\rho_{u}^{\mathrm{I}}$ and $\rho_{u}^{\mathrm{I}} X=\rho_{u}^{\mathrm{II}}$. If we set $X=\lambda=\rho_{u}^{\mathrm{II}} / \rho_{u}^{\mathrm{I}}$ then $\left(X_{x}, X_{u}\right) \neq$ $(0,0)$. This ensures existence of a function $U=U(t, x, u)$ which locally satisfies the equation $X_{x} U_{u}-X_{u} U_{x}=\rho_{u}^{\mathrm{I}}$. It is obvious that the so chosen functions $X$ and $U$ are functionally independent and that the above systems are then compatible with respect to $\Phi$ and $\Psi$, and hence the point transformation we sought for does exist.

Corollary 4. An equation $\mathcal{E}$ of the form (11) has (at least) two linearly independent conservation laws of density order 0 if and only if it can be locally reduced by a point transformation to the form

$$
\tilde{u}_{\tilde{t}}=D_{\tilde{x}}^{2} H\left(\tilde{t}, \tilde{x}, \tilde{u}_{0}, \ldots, \tilde{u}_{n-2}\right), \quad H_{\tilde{u}_{n-2}} \neq 0 .
$$


Proof. If $\mathcal{E}$ of the form (1) admits (at least) two linearly independent conservation laws of density order 0 , then by Theorem 2 we can assume (modulo a suitably chosen point transformation, if necessary) that $\mathcal{E}$ has the conservation laws $\mathcal{L}^{\mathrm{I}}$ and $\mathcal{L}^{\mathrm{II}}$ with the characteristics 1 and $x$, respectively. Then there exist conserved vectors $\left(\rho^{\mathrm{I}}, \sigma^{\mathrm{I}}\right) \in \mathcal{L}^{\mathrm{I}}$ and $\left(\rho^{\mathrm{II}}, \sigma^{\mathrm{II}}\right) \in \mathcal{L}^{\mathrm{II}}$ such that

$$
D_{t} \rho^{\mathrm{I}}+D_{x} \sigma^{\mathrm{I}}=u_{t}-F, \quad D_{t} \rho^{\mathrm{II}}+D_{x} \sigma^{\mathrm{II}}=x\left(u_{t}-F\right) .
$$

Up to the equivalence of conserved vectors modulo trivial ones we have $\rho^{\mathrm{I}}=u$ and $\rho^{\mathrm{II}}=x u$. Hence $D_{x} \sigma^{\mathrm{I}}=-F$ and $D_{x} \sigma^{\mathrm{II}}=-x F$. Combining these equalities, we find that $\sigma^{\mathrm{I}}=-D_{x}\left(\sigma^{\mathrm{II}}-\right.$ $\left.x \sigma^{\mathrm{I}}\right)$, i.e., $F=D_{x}^{2}\left(\sigma^{\mathrm{II}}-x \sigma^{\mathrm{I}}\right)$. As a result, we can represent the equation $\mathcal{E}$ in the form $u_{t}=D_{x}^{2} H$, where $H=\sigma^{\mathrm{II}}-x \sigma^{\mathrm{I}}$, ord $H=n-2$.

Conversely, assume that $\mathcal{E}$ is reduced to the equation $\tilde{u}_{\tilde{t}}=D_{\tilde{x}}^{2} H\left(\tilde{t}, \tilde{x}, \tilde{u}, \ldots, \tilde{u}_{n-2}\right)$, where $H_{\tilde{u}_{n-2}} \neq 0$, through a point transformation $\mathcal{T}$. The transformed equation $\tilde{u}_{\tilde{t}}=D_{\tilde{x}}^{2} H$ admits at least two linearly independent conservation laws, in particular, those with the characteristics 1 and $\tilde{x}$. Their preimages under $\mathcal{T}$ are linearly independent conservation laws of $\mathcal{E}$ whose density orders are zero, and the result follows.

Corollary 5. If an equation $\mathcal{E}$ of the form (1) with $n \geqslant 5$ (resp. $2 \leqslant n \leqslant 4$ ) has two linearly independent conservation laws $\mathcal{L}^{\mathrm{I}}$ and $\mathcal{L}^{\mathrm{II}}$ with $\operatorname{ord}_{\mathrm{d}} \mathcal{L}^{\mathrm{I}} \leqslant 1$ and $\operatorname{ord}_{\mathrm{d}} \mathcal{L}^{\mathrm{II}}<n / 2-1$ (resp. $\operatorname{ord}_{\mathrm{d}} \mathcal{L}^{\mathrm{I}}=\operatorname{ord}_{\mathrm{d}} \mathcal{L}^{\mathrm{II}}=0$ ), then the right-hand side $F$ of $\mathcal{E}$ has the form

$$
F=F_{3} u_{n}+F_{2} u_{n-1}^{2}+F_{1} u_{n-1}+F_{0},
$$

where $F_{0}, \ldots, F_{3}$ are differential functions of order less than $n-1$.

Remark 1. If in the proof of Corollary 4 we replace the conservation laws $\mathcal{L}^{\mathrm{I}}$ and $\mathcal{L}^{\mathrm{II}}$ by linear combinations thereof, $\hat{\mathcal{L}}^{\mathrm{I}}=a_{11} \mathcal{L}^{\mathrm{I}}+a_{12} \mathcal{L}^{\mathrm{II}}$ and $\hat{\mathcal{L}}^{\mathrm{II}}=a_{21} \mathcal{L}^{\mathrm{I}}+a_{22} \mathcal{L}^{\mathrm{II}}$, where $a_{i j}, i, j=1,2$, are arbitrary constants such that $a_{11} a_{22}-a_{12} a_{21} \neq 0$, then the associated equations of the form (16) are related through the transformation

$$
\hat{t}=\tilde{t}, \quad \hat{x}=\frac{a_{22} \tilde{x}+a_{21}}{a_{12} \tilde{x}+a_{11}}, \quad \hat{u}=\frac{\left(a_{12} \tilde{x}+a_{11}\right)^{3}}{a_{11} a_{22}-a_{12} a_{21}} \tilde{u}, \quad \hat{H}=\frac{a_{11} a_{22}-a_{12} a_{21}}{a_{12} \tilde{x}+a_{11}} H,
$$

where $\hat{t}, \hat{x}, \hat{u}$ and the differential function $\hat{H}$ correspond to the conservation laws $\hat{\mathcal{L}}^{\mathrm{I}, \mathrm{II}}$. Such transformations, considered for all admissible values of $a_{i j}, i, j=1,2$, form a subgroup $\mathcal{G}$ of the point equivalence group for the class of equations of the form (16). Thus, up to the $\mathcal{G}$-equivalence we can assume that the form (16) of the equation $\mathcal{E}$ is associated with the two-dimensional subspace spanned by its conservation laws $\mathcal{L}^{\mathrm{I}}$ and $\mathcal{L}^{\mathrm{II}}$ rather than with $\mathcal{L}^{\mathrm{I}}$ and $\mathcal{L}^{\mathrm{II}}$ per se.

\section{Examples: third-order evolution equations}

Example 1. We start with the so-called Harry Dym (HD) equation, see e.g. [13, Section 20.2] and references therein for more details:

$$
u_{t}=u^{3} u_{x x x}
$$

The subspace of its conservation laws of density order not greater than one is five-dimensional and generated by the zero-order conservation laws $\mathcal{L}^{i}, i=\mathrm{I}, \ldots, \mathrm{IV}$, with the densities $\rho^{\mathrm{I}}=u^{-2}$, $\rho^{\mathrm{II}}=x u^{-2}, \rho^{\mathrm{III}}=x^{2} u^{-2}$, and $\rho^{\mathrm{IV}}=u^{-1}$, and the first-order conservation law $\mathcal{L}^{V}$ with the density $\rho^{\mathrm{V}}=u_{x}^{2} u^{-1}$.

The first three densities agree in the sense that $\rho^{\mathrm{II}} / \rho^{\mathrm{I}}=\rho^{\mathrm{III}} / \rho^{\mathrm{II}}$. Hence upon introducing new variables $\tilde{t}=-2 t \operatorname{sign} u, \tilde{x}=x, \tilde{u}=u^{-2}$ obtained by applying Theorem 2 to $\mathcal{L}^{\mathrm{I}}$ and $\mathcal{L}^{\mathrm{II}}$, the HD equation can be rewritten in an even more specific than (16), and also well-known, conservative 
form $\tilde{u}_{\tilde{t}}=D_{\tilde{x}}^{3}\left(\tilde{u}^{-1 / 2}\right)$. (We transformed $t$ above in order to simplify the transformed equation.) The transformed equation obviously admits conservation laws with the characteristics equal to $1, x$ and $x^{2}$.

For the pair of conservation laws $\mathcal{L}^{\mathrm{IV}}$ and $\mathcal{L}^{\mathrm{I}}$ Theorem 2 yields the transformation $\tilde{t}=t$, $\tilde{x}=-2 / u, \tilde{u}=x / 2$ which maps the HD equation into the equation

$$
\tilde{u}_{\tilde{t}}=D_{\tilde{x}}^{2}\left(\frac{1}{2 \tilde{x}^{3} \tilde{u}_{\tilde{x}}^{2}}\right)
$$

The conservation law $\mathcal{L}^{\mathrm{V}}$ is mapped into a conservation law with the characteristic 1 by the contact transformation $\tilde{t}=t, \tilde{x}=u_{x}^{2} / u, \tilde{u}=u-2 u / u_{x}, \tilde{u}=u^{2} / u_{x}^{3}$ constructed using the method from the proof of Theorem 1. The corresponding transformed equation reads

$$
\tilde{u}_{\tilde{t}}=D_{\tilde{x}}\left(\frac{-\tilde{x}^{8} \tilde{u}_{\tilde{x}}^{6}}{4\left(2 \tilde{x} \tilde{u}_{\tilde{x} \tilde{x}}+3 \tilde{u}_{\tilde{x}}\right)^{2}}\right)
$$

Example 2. Consider now the class of KdV-type equations

$$
u_{t}=u_{x x x}+f(u) u_{x} .
$$

Any equation from this class admits at least three conservation laws $\mathcal{L}^{i}, i=\mathrm{I}, \ldots, \mathrm{III}$, with the densities $\rho^{\mathrm{I}}=u, \rho^{\mathrm{II}}=u^{2} / 2, \rho^{\mathrm{III}}=-u_{x}^{2} / 2+\check{f}(u)$, where $\partial \hat{f} / \partial u=f, \partial \check{f} / \partial u=\hat{f}$. It is straightforward to verify that if $\partial^{3} f / \partial u^{3} \neq 0$ these conservation laws form a basis in the space of the conservation laws of density order not greater than one.

The reduction (17) to the form (14) using $\mathcal{L}^{\mathrm{I}}$ (resp. $\mathcal{L}^{\mathrm{II}}$ ) according to Theorem 1 is immediate. The conservation law $\mathcal{L}^{\mathrm{I}}$ gives rise to the identity transformation and the representation $u_{t}=$ $D_{x}\left(u_{x x}+\hat{f}(u)\right)$ for (17). The transformation associated with $\mathcal{L}^{\mathrm{II}}$ is $\tilde{t}=t, \tilde{x}=x$ and $\tilde{u}=\rho^{\mathrm{II}}=$ $u^{2} / 2$. It maps equation (17) into

$$
\tilde{u}_{\tilde{t}}=D_{\tilde{x}}\left(\tilde{u}_{\tilde{x} \tilde{x}}-\frac{3}{4} \frac{\tilde{u}_{\tilde{\tilde{u}}}^{2}}{\tilde{u}}+\varepsilon \sqrt{2 \tilde{u}} \hat{f}(\varepsilon \sqrt{2 \tilde{u}})-\check{f}(\varepsilon \sqrt{2 \tilde{u}})\right)
$$

where $\varepsilon=\operatorname{sign} u$.

Now consider the conservation laws $\mathcal{L}^{\mathrm{I}}$ and $\mathcal{L}^{\mathrm{II}}$ and apply Theorem 2, We can directly follow the procedure from the proof of this theorem and set $\tilde{t}=t, \tilde{x}=\rho_{u}^{\mathrm{II}}=u$ and $\tilde{u}=x$. This is nothing but the hodograph transformation interchanging $x$ and $u$. It reduces equation (17) to the equation (cf. [1] $)$

$$
\tilde{u}_{\tilde{t}}=D_{\tilde{x}}^{2}\left(\frac{1}{2 \tilde{u}_{\tilde{x}}^{2}}-\check{f}(\tilde{x})\right)
$$

Example 3. The KdV equation, i.e., equation (17) with $f(u)=u$, possesses one more linearly independent zero-order conservation law $\mathcal{L}^{\mathrm{IV}}$ with the density $\rho^{\mathrm{IV}}=x u+t u^{2} / 2$, cf. [21]. This gives more possibilities for reduction to the forms (14) and (16).

In analogy with the previous example, we find that the transformation associated with $\mathcal{L}^{\mathrm{IV}}$ is $\tilde{t}=t, \tilde{x}=x$ and $\tilde{u}=\rho^{\mathrm{IV}}=x u+t u^{2} / 2$. It maps the $\mathrm{KdV}$ equation into

$$
\tilde{u}_{\tilde{t}}=D_{\tilde{x}}\left(\tilde{u}_{\tilde{x} \tilde{x}}-\frac{3}{2} \frac{\tilde{t}}{Z} \tilde{u}_{\tilde{x}}^{2}-3 \frac{\tilde{x}}{Z} \tilde{u}_{\tilde{x}} \pm \frac{Z^{3 / 2}}{3 \tilde{t}^{2}}+3 \frac{\tilde{u}}{Z}-\frac{\tilde{x}}{\tilde{t}} \tilde{u}-\frac{\tilde{x}^{3}}{3 \tilde{t}^{2}}\right),
$$

where $Z=\tilde{x}^{2}+2 \tilde{t} \tilde{u}$. 
For the pair of the conservation laws $\mathcal{L}^{\mathrm{I}}$ and $\mathcal{L}^{\mathrm{IV}}$ we have the transformation of the form $\tilde{t}=t, \tilde{x}=\rho_{u}^{\mathrm{IV}}=x+t u$ and $\tilde{u}=u$, and the transformed equation reads

$$
\tilde{u}_{\tilde{t}}=D_{\tilde{x}}\left(\frac{\tilde{u}_{\tilde{x} \tilde{x}}}{\left(1-t \tilde{u}_{\tilde{x}}\right)^{3}}\right)=D_{\tilde{x}}^{2}\left(\frac{\left(1-t \tilde{u}_{\tilde{x}}\right)^{-2}}{2 t}\right) .
$$

Another pair of the conservation laws, $\mathcal{L}^{\mathrm{II}}$ and $\mathcal{L}^{\mathrm{IV}}$, gives rise to a more complicated transformation $\tilde{t}=t, \tilde{x}=x / u+t, \tilde{u}=u^{3} / 3$, and a more cumbersome transformed equation,

$$
\tilde{u}_{\tilde{t}}=D_{\tilde{x}}^{2}\left(\frac{\left((\tilde{x}-\tilde{t}) \tilde{u}_{\tilde{x}}+6 \tilde{u}\right) \tilde{u}_{\tilde{x}}}{2\left((\tilde{x}-\tilde{t}) \tilde{u}_{\tilde{x}}+3 \tilde{u}\right)^{2}}\right) .
$$

Note that exhaustive lists of one- and two-dimensional subspaces of zero-order conservation laws of the $\mathrm{KdV}$ equation that are not equivalent with respect to the Lie point symmetry group of the latter are $\left\{\left\langle\mathcal{L}^{\mathrm{I}}\right\rangle,\left\langle\mathcal{L}^{\mathrm{II}}\right\rangle,\left\langle\mathcal{L}^{\mathrm{IV}}\right\rangle\right\}$ and $\left\{\left\langle\mathcal{L}^{\mathrm{I}}, \mathcal{L}^{\mathrm{II}}\right\rangle,\left\langle\mathcal{L}^{\mathrm{I}}, \mathcal{L}^{\mathrm{IV}}\right\rangle,\left\langle\mathcal{L}^{\mathrm{II}}, \mathcal{L}^{\mathrm{IV}}\right\rangle\right\}$, respectively. Therefore, the above description of normal forms (14) and (16) related to zero-order conservation laws of the $\mathrm{KdV}$ equation is complete modulo the action of the Lie point symmetry group of the KdV equation, cf. Remark 1.

Example 4. The Schwarzian KdV equation

$$
u_{t}=u_{x x x}-\frac{3}{2} \frac{u_{x x}^{2}}{u_{x}}
$$

possesses no zero-order conservation laws. The subspace of its first-order conservation laws is spanned by the conservation laws $\mathcal{L}^{i}, i=\mathrm{I}, \ldots, \mathrm{III}$, with the densities $\rho^{\mathrm{I}}=1 / u_{x}, \rho^{\mathrm{II}}=u / u_{x}$, $\rho^{\mathrm{III}}=u^{2} / u_{x}$. For transforming $\mathcal{L}^{\mathrm{I}}$ into a conservation law with the density $u$, we construct, following the proof of Theorem 1, the contact transformation

$$
\tilde{t}=t, \quad \tilde{x}=u_{x}, \quad \tilde{x}=\frac{2 x}{u_{x}^{2}}-\frac{2 u}{u_{x}^{3}}, \quad \tilde{u}=-\frac{4 x}{u_{x}^{3}}+\frac{6 u}{u_{x}^{4}},
$$

which maps the Schwarzian KdV equation into

$$
\tilde{u}_{\tilde{t}}=D_{\tilde{x}}\left(\frac{-4 \tilde{x}^{-5}}{\left(\tilde{x}^{2} \tilde{u}_{\tilde{x} \tilde{x}}+6 \tilde{x} \tilde{u}_{\tilde{x}}+6 \tilde{u}\right)^{2}}\right) \text {. }
$$

Further examples of normal forms for low-order nonlinear evolution equations, including physically relevant examples like the nonlinear diffusion-convection equations, can be found in 28 .

\section{Conservation laws of linear evolution equations}

Any linear partial differential equation admits conservation laws whose characteristics depend on independent variables only and run through the set of solutions of the adjoint equation. The corresponding conserved vectors are linear with respect to the unknown function and its derivatives. It is natural to call the conservation laws of this kind linear [24, Section 5.3]. Let us stress that, following the literature, here and below we allow for a slight abuse of terminology by calling a conservation law linear (resp. quadratic) when it contains a conserved vector which is linear (resp. quadratic) in the totality of variables $u_{0}, u_{1}, u_{2}, \ldots$

The problem of describing other kinds of conservation laws for general linear partial differential equations is quite difficult. However, it can be solved for certain special classes of equations including linear $(1+1)$-dimensional evolution equations. 
Consider an equation $\mathcal{E}$ of form (1), where the function $F$ is linear in $u_{0}, \ldots, u_{n}$, i.e.,

$$
F=\mathfrak{F} u=\sum_{i=0}^{n} A^{i}(t, x) u_{i}, \quad \text { where } \quad \mathfrak{F}=\sum_{i=0}^{n} A^{i}(t, x) D_{x}^{i}, \quad A^{n} \neq 0 .
$$

Thus, the equation $\mathcal{E}$ reads

$$
u_{t}=\mathfrak{F} u \text {. }
$$

Then the condition (10) for cosymmetries takes the form

$$
D_{t} \gamma+\mathfrak{F}^{\dagger} \gamma=0 \bmod \check{\mathcal{E}}, \quad \text { where } \quad \mathfrak{F}^{\dagger}=\sum_{i=0}^{n}\left(-D_{x}\right)^{i} \circ A^{i}(t, x) .
$$

The operator $\mathfrak{F}^{\dagger}$ is the formal adjoint of $\mathfrak{F}$. Writing out the condition (10) yields

$$
\gamma_{t}+\sum_{k} \gamma_{u_{k}} \sum_{i=0}^{n} \sum_{j=0}^{k}\left(\begin{array}{l}
k \\
j
\end{array}\right) A_{k-j}^{i} u_{i+j}+\sum_{i=0}^{n}(-1)^{i} \sum_{s=0}^{i}\left(\begin{array}{l}
i \\
s
\end{array}\right) A_{i-s}^{i} D_{x}^{s} \gamma=0,
$$

where $A_{j}^{i}=\partial^{j} A^{i} / \partial x^{j}$.

A function $v=v(t, x)$ is a cosymmetry of the equation $\mathcal{E}$ if and only if it is a solution of the adjoint equation $\mathcal{E}^{*}$ :

$$
v_{t}+\mathfrak{F}^{\dagger} v=0
$$

Any cosymmetry of $\mathcal{E}$ that does not depend on $u$ and the derivatives thereof is a characteristic of a linear conservation law of $\mathcal{E}$, and any linear conservation law of $\mathcal{E}$ has a characteristic of this form. Namely, a solution $v=v(t, x)$ of the adjoint equation $\mathcal{E}^{*}$ corresponds to the conserved vector $(\rho, \sigma)$ of $\mathcal{E}$ with $\rho=v(t, x) u$ and $\sigma=\sum_{i=0}^{n-1} \sigma^{i}(t, x) u_{i}$. The coefficients $\sigma^{i}$ are found recursively from the equations

$$
\sigma^{n-1}=-v A^{n}, \quad \sigma^{i}=-v A^{i+1}-\sigma_{x}^{i+1}, \quad i=n-2, \ldots, 0 .
$$

It turns out that all cosymmetries of even-order equations (18) are of this form.

Theorem 3. For any linear $(1+1)$-dimensional evolution equation of even order, all its cosymmetries depend only on $x$ and $t$, and the space of all cosymmetries is isomorphic to the solution space of the associated adjoint equation.

Proof. Suppose that there exists a $\nu \in \mathbb{N} \cup\{0\}$ such that $\gamma_{u_{\nu}} \neq 0$ and denote

$$
r=\max \left\{\nu \in \mathbb{N} \cup\{0\} \mid \gamma_{u_{\nu}} \neq 0\right\} .
$$

For even $n$ vanishing of the coefficient at $u_{n+r}$ in (19) yields the equation $2 A^{n} \gamma_{u_{r}}=0$, whence $\gamma_{u_{r}}=0$. This contradicts the original assumption, and hence $\gamma$ depends only on $t$ and $x$.

Corollary 6. For any linear $(1+1)$-dimensional evolution equation of even order its space of conservation laws is exhausted by linear ones and is isomorphic to the solution space of the corresponding adjoint equation.

For odd $n$ things become somewhat more involved.

Theorem 4. For any linear $(1+1)$-dimensional evolution equation of odd order, all its cosymmetries are affine in the totality of variables $u_{0}, u_{1}, u_{2}, \ldots$ 
Proof. In contrast with the case of even $n$, now the coefficient at $u_{n+r}$ in (19) vanishes identically. Requiring the coefficient at $u_{n+r-1}$ in (19) to vanish yields

$$
n A_{0}^{n} D_{x} \gamma_{u_{r}}=\left(2 A_{0}^{n-1}+(r-1-n) A_{1}^{n}\right) \gamma_{u_{r}},
$$

so $\gamma_{u_{r}}$ depends only on $t$ and $x$. Using this result while evaluating the coefficient at $u_{n+r-2}$ yields

$$
n A_{0}^{n} D_{x} \gamma_{u_{r-1}}=\left(2 A_{0}^{n-1}+(r-1-n) A_{1}^{n}\right) \gamma_{u_{r-1}}+\psi^{r-1},
$$

where $\psi^{r-1}$ is a function of $t$ and $x$, which is expressed via $\gamma_{u_{r}}$ and $A^{i}$; the explicit form of $\psi^{r-1}$ is not important here. Thus, $\gamma_{u_{r-1}}$ also depends only on $t$ and $x$. Iterating the above procedure allows us to conclude that the function $\gamma$ is affine in $u_{0}, \ldots, u_{r}$, that is,

$$
\gamma=\Gamma u+v(t, x), \quad \Gamma=\sum_{k=0}^{r} g^{k}(t, x) D_{x}^{k},
$$

and the result follows.

Note that if we restrict ourselves to the case of polynomial cosymmetries for equations with constant coefficients, then upon combining Theorems 3 and 4 we recover (part of) Proposition 1 of $[12$.

For the equations (18) with $\mathfrak{F}^{\dagger}=-\mathfrak{F}$ the determining equations for cosymmetries and for characteristics of generalized symmetries coincide. This observation in conjunction with Theorem 4 implies the following assertion (cf. [33]).

Corollary 7. For any linear $(1+1)$-dimensional evolution equation (18) of odd order such that $\mathfrak{F}^{\dagger}=-\mathfrak{F}$, all its generalized symmetries are affine in $u_{j}$ for all $j$.

Now let us get back to the general case of (18) with odd $n$. Substituting the representation (22) for $\gamma$ into (19) reveals that $v=v(t, x)$ satisfies the adjoint equation (20) which is decoupled from the equations for $g^{i}$. Thus, $v$ is a cosymmetry per se. Just as before, to any such cosymmetry there corresponds a linear conservation law with the density $\rho=v(t, x) u$. However, the issue of existence of conservation laws associated with cosymmetries linear in $u_{j}$ is nontrivial.

Indeed, let $\gamma=\Gamma u$. As we want $\gamma$ to be a characteristic of a conservation law, we should require that $\gamma \in \operatorname{Im} \delta / \delta u$ (cf. Section 3 ). Hence, the operator $\Gamma$ should be formally self-adjoint and, in particular, its order should be even (note, however, that if $\Gamma$ is not formally self-adjoint, we can take its formally self-adjoint part $\tilde{\Gamma}=\left(\Gamma+\Gamma^{\dagger}\right) / 2 ; \tilde{\gamma}=\tilde{\Gamma} u$ is easily verified to be a cosymmetry if so is $\gamma$ ). The density of the conservation law associated with the characteristic $\gamma$ reads, up to the usual addition of a total $x$-derivative of something, $\rho=\frac{1}{2} u \Gamma u$. Without loss of generality we can also assume the corresponding flux to be quadratic in $u_{0}, u_{1}, \ldots$, see Theorem 5.104 of [24], so the conservation law in question is quadratic, and we obtain the following result.

Theorem 5. For any linear $(1+1)$-dimensional evolution equation of odd order, the space of its conservation laws is spanned by linear and quadratic ones.

For linear conservation laws with the densities of the form $\rho=v(t, x) u$ where $v$ solves the adjoint equation we still have (21).

Now turn to the quadratic conservation laws. The differential function $\Gamma u$ is a characteristic of a conservation law for $\mathcal{E}$ if and only if the operator $\Gamma$ satisfies the following equivalent conditions:

1) it maps the solutions of the equation $\mathcal{E}$ into solutions of the adjoint equation $\mathcal{E}^{*}$;

2) $\partial \Gamma / \partial t+\Gamma \mathfrak{F}+\mathfrak{F}^{\dagger} \Gamma=0$

3) $\left(\partial_{t}+\mathfrak{F}^{\dagger}\right) \Gamma=\Gamma\left(\partial_{t}-\mathfrak{F}\right)$, i.e., the operator $\Gamma\left(\partial_{t}-\mathfrak{F}\right)$ is formally skew-adjoint. 
Note that if the operator $\mathfrak{F}$ is formally skew-adjoint $\left(\mathfrak{F}^{\dagger}=-\mathfrak{F}\right)$ then the operators $\partial_{t}-\mathfrak{F}$ and $\Gamma$ commute: $\left[\partial_{t}-\mathfrak{F}, \Gamma\right]=0$, i.e., $\Gamma$ is a symmetry operator for the equation $\mathcal{E}$.

Any linear equation admits a symmetry $u \partial_{u}$, and the associated operator $\Gamma$ is the identity operator which is obviously formally self-adjoint. Combining this result with the above we obtain the following assertion.

Proposition 2. Any linear $(1+1)$-dimensional evolution equation (18) of odd order such that $\mathfrak{F}^{\dagger}=-\mathfrak{F}$ possesses a conservation law with the density $\rho=u^{2}$.

Moreover, linear $(1+1)$-dimensional evolution equations of odd order can possess infinite series of quadratic conservation laws of arbitrarily high orders, as illustrated by the following example.

Example 5. Consider the equation

$$
u_{t}=u_{x x x} .
$$

It is straightforward to verify that in this case the determining equations for cosymmetries and characteristics of (generalized) symmetries coincide because Eq. (23) is identical with its adjoint.

Denote by $\mathcal{S}$ the space of all generalized symmetries of (23) and let $\mathcal{Q}$ be the space of symmetries of the form $f(t, x) \partial_{u}$, where $f$ solves (23) : $f_{t}=f_{x x x}$. By Corollary 7 the quotient space $\mathcal{S} / \mathcal{Q}$ is exhausted by linear generalized symmetries. Successively solving the determining equations (cf. e.g. [23]) we find that the space $\mathcal{S} / \mathcal{Q}$ is spanned by the symmetries of the form $\left(D_{x}^{k} \Upsilon^{l} u\right) \partial_{u}$, where $k, l=0,1,2, \ldots$ and $\Upsilon=x+3 t D_{x}^{2}$.

As the determining equations for symmetries and cosymmetries of (23) coincide, the space of cosymmetries for (23) is spanned by the following objects:

1 ) the cosymmetries of the form $f(t, x)$ where $u=f(t, x)$ is any solution of (23);

2) the cosymmetries of the form $D_{x}^{k} \Upsilon^{l} u$, where $k, l=0,1,2, \ldots$

Any cosymmetry of the first kind is associated with a conservation law with the density $\rho=f(t, x) u$. As for cosymmetries of the second kind, only those with even $k=2 m$ are characteristics of the conservation laws. The conservation laws in question can (modulo trivial ones) be chosen to be quadratic, with the densities $\rho_{l m}=\frac{1}{2} u D_{x}^{m} \Upsilon^{l} D_{x}^{m} u$ and the density orders $l+m, l, m=0,1,2 \ldots$

However, there also exist linear $(1+1)$-dimensional evolution equations of odd order which have no quadratic conservation laws.

Example 6. The operator $\mathfrak{F}=D_{x}^{3}+x$ associated with the equations

$$
u_{t}=u_{x x x}+x u
$$

is not formally skew-adjoint. Equation (24) possesses nontrivial symmetries which are linear combinations of the operators $\left(\left(D_{x}^{3}+x\right)^{k}\left(D_{x}+t\right)^{l} u\right) \partial_{u}$ but they cannot be employed for construction of quadratic conservation laws of (24) in the above fashion.

In fact, all cosymmetries of (24) depend only on $x$ and $t$, and therefore this equation has no quadratic conservation laws.

Indeed, using the proof by contradiction, suppose that (24) has a cosymmetry $\gamma=\Gamma u$, and ord $\gamma=r$, i.e., $g^{r} \neq 0$. The condition $\partial \Gamma / \partial t+\Gamma \mathfrak{F}+\mathfrak{F}^{\dagger} \Gamma=0$ implies the following system of determining equations for the coefficients of $\Gamma$ :

$$
\begin{aligned}
& 3 g_{x}^{i}=(i+3) g^{i+3}+g_{t}^{i+2}-g_{x x x}^{i+2}+2 x g^{i+2}-3 g_{x x}^{i+1}, \quad i=1, \ldots, r, \\
& 2 g^{2}+g_{t}^{1}-g_{x x x}^{1}+2 x g^{1}-3 g_{x x}^{0}=0, \\
& g^{1}+g_{t}^{0}-g_{x x x}^{0}+2 x g^{0}=0,
\end{aligned}
$$


where the functions $g^{r+3}, g^{r+2}$ and $g^{r+1}$ vanish by definition. We successively integrate (25) starting from the equations with the greatest value of $i$ and going down. The equations for $i=r$ and $i=r-1$ imply that the coefficients $g^{r}$ and $g^{r-1}$ depend on $t$ but not on $x$. Proceeding by induction, we find that for any $j=0, \ldots, r$ the function $g^{r-j}$ is a polynomial in $x$ of degree $2[j / 2]$. The ratio of the coefficient at the highest power of $x$ in $g^{r-j}$ to $g^{r}$ (resp. $g^{r-1}$ ) is a constant if $j$ is even (resp. odd). Then (26) and (27) imply $g^{r}=0$ and $g^{r-1}=0$. This contradicts our assumption that $g^{r} \neq 0$, and the result follows.

\section{Conclusions}

In this paper we have presented normal forms for the evolution equations in two independent variables possessing low-order conservation laws, see Theorems 1 and 2 , and Corollaries 25 for details. Using these normal forms considerably simplifies the construction of nonlocal variables associated with the conservation laws in question and hence the study of the Abelian coverings and nonlocal symmetries, including potential symmetries, for the equations in question in spirit of [7, 25, 27, 16, 32], and references therein. As these normal forms are associated, up to a certain natural equivalence (see Remark 1), with the subspaces spanned by conservation laws rather than conservation laws per se, we are naturally led to pose the problem of classification of inequivalent subspaces of (low-order) conservation laws for the classes or special cases of evolution equations of interest.

As for the linear evolution equations in two independent variables, we have shown that their conservation laws are (modulo trivial conserved vectors, of course) at most quadratic in the dependent variable and the derivatives thereof, see Theorem 3. Moreover, for the linear evolution equations of even order their conservation laws are at most linear in these quantities, and the associated densities can be chosen to have the form of a product of the dependent variable with a solution of the adjoint equation (Theorem 4). It is natural to ask whether similar results can be obtained for more general linear PDEs (cf. [33] for the case of symmetries), and we intend to address this issue in our future work.

\section{Acknowledgements}

The research of R.O.P. was supported by the project P20632 of the Austrian Science Fund. The research of A.S. was supported in part by the Ministry of Education, Youth and Sports of the Czech Republic (MŠMT ČR) under grant MSM 4781305904, and by Silesian University in Opava under grant IGS 2/2009. The authors are pleased to thank M. Kunzinger for stimulating discussions. A.S. gratefully acknowledges the warm hospitality extended to him by the Department of Mathematics of the University of Vienna during his visits in the course of preparation of the present paper.

It is our great pleasure to thank the referees for useful suggestions that have considerably improved the paper.

\section{References}

[1] Abellanas L. and Galindo A., Conserved densities for nonlinear evolution equations. I. Even order case, J. Math. Phys. 20 (1979), 1239-1243.

[2] Abellanas L. and Galindo A., Conserved densities for nonlinear evolution equations. II. Odd order case, J. Math. Phys. 22 (1981), 445-448.

[3] Abellanas L. and Galindo A., Conserved densities for linear evolution systems, Comm. Math. Phys. 79 (1981), 341-351. 
[4] Abellanas L. and Galindo A., Evolution equations with high order conservation laws, J. Math. Phys. 24 (1983), 504-509.

[5] Ashton A.C.L., Conservation laws and non-Lie symmetries for linear PDEs, J. Nonlin. Math. Phys. 15 (2008), 316-332.

[6] Błaszak M., Multi-Hamiltonian theory of dynamical systems, Springer-Verlag, Berlin, 1998.

[7] Bocharov A.V., Chetverikov V.N., Duzhin S.V., Khor'kova N.G., Krasil'shchik I.S., Samokhin A.V., Torkhov Yu.N., Verbovetsky A.M., and Vinogradov A.M., Symmetries and conservation laws for differential equations of mathematical physics, American Mathematical Society, Providence, RI, 1999.

[8] Bryant R.L. and Griffiths P.A., Characteristic cohomology of differential systems II: Conservation laws for a class of parabolic equations, Duke Math. J. 78 (1995), 531-676.

[9] Drinfel'd V.G., Svinolupov S.I., Sokolov V.V., Classification of fifth-order evolution equations having an infinite series of conservation laws, Dokl. Akad. Nauk Ukrain. SSR Ser. A 1985, no. 10, 8-10 (in Russian).

[10] Foltinek K., Conservation laws of evolution equations: generic non-existence, J. Math. Anal. Appl. 235 (1999), 356-379.

[11] Foltinek K., Third-order scalar evolution equations with conservation laws, Selecta Math. (N.S.) 8 (2002), 201-235.

[12] Galindo A., Constants of motion for linear evolution systems, Lett. Math. Phys. 5 (1981), 293-301.

[13] Ibragimov N.H., Transformation groups applied to mathematical physics, D. Reidel Publishing Co., Dordrecht, 1985.

[14] Igonin S., Conservation laws for multidimensional systems and related linear algebra problems, J. Phys. A: Math. Gen. 35 (2002), 10607-10617.

[15] Kaptsov O.I., Classification of evolution equations by conservation laws, Funct. Anal. Appl. 16 (1982), 61-63.

[16] Kunzinger M. and Popovych R.O., Potential conservation laws, J. Math. Phys. 49 (2008), 103506, 34 pp.; arXiv:0803.1156.

[17] Magadeev B.A., On group classification of nonlinear evolution equations, Algebra i Analiz 5 (1993), 141-156 (in Russian); English translation in St. Petersburg Math. J. 5 (1994), 345-359.

[18] Mikhailov A.V., Novikov V.S., and Wang J.P., Symbolic representation and classification of integrable systems, in Algebraic theory of differential equations, M.A.H. MacCallum and A.V. Mikhailov eds., Cambridge University Press, Cambridge, 2009, pp. 156-216.

[19] Mikhailov A.V., Shabat A.B. and Sokolov V.V., Symmetries of differential equations and the problem of integrability, in What is integrability?, ed. by V.E. Zakharov, Springer, Berlin, 1991, pp. 115-184.

[20] Mikhailov A.V. and Sokolov V.V., Symmetries of differential equations and the problem of integrability, in Integrability, ed. by A.V. Mikhailov, Springer, Berlin-Heidelberg, 2009, pp. 19-98.

[21] Miura R.M., Gardner C.S., and Kruskal M.D., Korteweg-de Vries equation and generalizations. II. Existence of conservation laws and constants of motion, J. Math. Phys. 9 (1968), 1204-1209.

[22] Newell A.C., Solitons in mathematics and physics, Society for Industrial and Applied Mathematics (SIAM), Philadelphia, PA, 1985.

[23] Nikitin A.G., Complete set of symmetry operators of the Schrödinger equation, Ukrainian Math. J. 43 (1991), 1413-1418.

[24] Olver P.J., Applications of Lie groups to differential equations, Springer-Verlag, New York, 1993.

[25] Popovych R.O. and Ivanova N.M., Hierarchy of conservation laws of diffusion-convection equations, J. Math. Phys. 46 (2005), 043502, 22 pp.; arXiv:math-ph/0407008

[26] Popovych R.O., Kunzinger M., and Eshraghi H., Admissible point transformations of nonlinear Schrodinger equations, Acta Appl. Math. 109 (2010), 315-359; arXiv:math-ph/0611061.

[27] Popovych R.O., Kunzinger M. and Ivanova N.M., Conservation laws and potential symmetries of linear parabolic equations, Acta Appl. Math. 100 (2008), 113-185; arXiv:0706.0443.

[28] Popovych R.O. and Samoilenko A.M., Local conservation laws of second-order evolution equations, J. Phys. A 41 (2008), 362002, 11 pp.; arXiv:0806.2765

[29] Sanders J.A. and Wang J.P., Classification of conservation laws for KdV-like equations, Math. Comput. Simulation 44 (1997), 471-481. 
[30] Sanders J.A. and Wang J.P., The symbolic method and cosymmetry integrability of evolution equations, in International Conference on Differential Equations (Berlin, 1999), World Sci. Publ., River Edge, NJ, 2000, Vol. 2, pp. 824-831.

[31] Sanders J.A. and Wang J.P., Number theory and the symmetry classification of integrable systems, in Integrability, A.V. Mikhailov ed., Springer, Berlin, 2008, pp. 89-118.

[32] Sergyeyev A., On recursion operators and nonlocal symmetries of evolution equations, in Proceedings of the Seminar on Differential Geometry, Silesian University in Opava, Opava, 2000, pp. 159-173; arXiv:nlin/0012011.

[33] Shapovalov A.V. and Shirokov I.V., Symmetry algebras of linear differential equations, Theoret. and Math. Phys. 92 (1992), 697-703.

[34] Svinolupov S.I. and Sokolov V.V., Evolution equations with nontrivial conservation laws, Funct. Anal. Appl. 16 (1982), 317-319. 